\title{
The Use of two Mesenchymal Stem Cell Doses as a Treatment for a Horse with Superficial Digital Flexor Tendinopathy
}

\author{
Miroslav Trunda, Sarka Vosahlikova, Klara Prajzlerova, Marika Stepankova, \\ Josef Bodor and Radek Klubal
}

Ceska geneticka banka spol. s r.o., Praha, Czech Republic, Prague

Correspondence should be addressed to: Miroslav Trunda; miroslav.trunda@genetickabanka.cz,

Received 16 May 2013; Accepted 10 June 2013; Published 29 August 2013

Academic Editor: Giacomo F. Rossi

Copyright (C) 2013 Miroslav Trunda, Sarka Vosahlikova, Klara Prajzlerova, Marika Stepankova, Josef Bodor and Radek Klubal. Distributed under Creative Commons CC-BY 3.0

\begin{abstract}
A clinical examination and ultrasonograph of an eight year old gelding revealed tendinopathy of a superficial digital flexor tendon (SDFT) after an injury in a cross-country race. The horse was treated using ex vivo-expanded autologous mesenchymal stem cells (MSCs) derived from bone marrow because of their immunoregulatory properties and their ability to heal cartilage and tendon under specific inflammatory conditions, such as trauma. The first application of MSCs into the lesion was performed on day 37 after the injury, and the second one on day 60. After the treatment with MSCs, the tissue of the injured tendon was nearly completely healed on day 120 after the injury. The horse started to compete in international eventing three months later, once again reaching a similar standard of performance.
\end{abstract}

Keywords: Mesenchymal stem cells (MSCs), horse, tendonitis, bone marrow.

\section{Introduction}

Tendinopathy or tendonitis remains a catastrophic injury among race horses. There are various methods routinely used for the treatment of this injury; for example, the transfer of autologous conditioned plasma (ACP) or platelet rich plasma (PRP). Furthermore, desmotomy of the accessory ligament of the superficial digital flexor tendon (SDFT), is an option to conservative therapy $\left[\begin{array}{lll}1, & 2,3\end{array}\right]$. Nevertheless, the main caveat using these treatments is a prolonged convalescence of the horse accompanied by at least one year out of training and racing. Smith et al., were the first to use autologous bone marrow mesenchymal stem cells (MSCs) to treat spontaneous tendon lesions in horses [4]. MSCs have been recently explored as a powerful tool to accelerate and improve healing in the treatment of tendonitis [6]. Because of their immunoregulatory properties MSCs also regenerate cartilage and tendon under specific inflammatory conditions, such as trauma [5]. After the expansion autologous, MSCs were reintroduced into the lesions of the injured tendon of the horse in two applications and shown to improve and accelerate healing. The aim of this study is to demonstrate the beneficial effect of the second application of autologous ex vivo-expanded MSCs

Cite this Article as: Miroslav Trunda, Sarka Vosahlikova, Klara Prajzlerova, Marika Stepankova, Josef Bodor and Radek Klubal (2013), "The Use of two Mesenchymal Stem Cell Doses as a Treatment for a Horse with Superficial Digital Flexor Tendinopathy," International Journal of Veterinary Medicine: Research \& Reports, Vol. 2013 (2013), Article ID 703351, DOI: 10.5171/2013.703351 
derived from bone marrow in the healing of severe tendonitis of SDFT.

\section{Clinical case}

\section{The Animal and its History}

An eight year old gelding (warmblood) was actively participating in three day eventing. On the day of the injury (day 0) swelling, pain, and fever in the front left limb were observed. Non-steroidal anti-inflammatory drug (NSAID) $\quad(1.1 \mathrm{mg} / \mathrm{kg}$ flunixin meglumine) was administered for 6 days (first two days i.v., following four days p.o.). The horse has not had any prior injury.

\section{Ultrasonographic Examination}

Tendonitis of SDFT was diagnosed by ultrasound examinationon day 20 after the injury. The injured tendon was examined using an ultrasonographic 7.5 $\mathrm{MHz}$ linear probe (Medison SonoAce 600). This examination was performed on the distal metacarpus of the horse transversally and longitudinally. The lesion was localized in the middle of SDFT without any visible surface abrasion. There was no open injury detected. The extent of injured lesions (Fig. 1) was estimated to be approximately $70 \%$ of healthy tendon tissue.

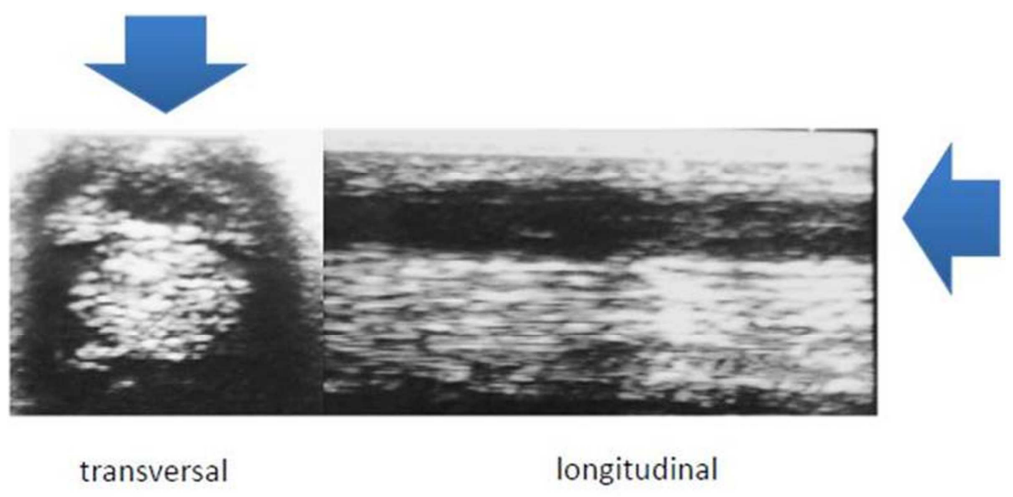

Fig. 1. Ultrasound Examination of Injured SDFT (Distal Metacarpus of the Horse)
Transversal and Longitudinal Projectionson Day 20 after the Injury. Arrows Point
towards Injured Lesions Depicted in Both Projections.

\section{Flow Analysis}

The staining of surface molecules was performed using direct fluorochromeconjugated monoclonal antibodies. The number, viability, and expression of clusters of differentiation (CD) markers characterizing MSCs (CD44, CD90, CD105) were acquired on FACS Canto (BD Biosciences) and analyzed using Diva6 software (BD Biosciences).

\section{Therapy}

\section{Bone Marrow Collection}

Collection of bone marrow (day 20 after the injury) was performedon the sedated horse (using $\alpha 2$ adrenergic agonist detomidine hydrochloride $(0,02 \mathrm{mg} / \mathrm{kg})$, and opioid analgesic butorphanol $\quad 0,025$ $\mathrm{mg} / \mathrm{kg}$ )). The Tuber coxae was shaved, desinfected, and a local anesthetic was injected (mepivacaine hydrochloride). Bone marrow (approximately 20ml) was extracted by aspiration biopsy using a $14 \mathrm{G}$ needle (14G/2.1mm; 55mm SOMATEX) into a syringe containing heparin $(0.6 \mathrm{ml}$ $5,000 \mathrm{IU})$.

\section{Preparation of MSCs}

The mononuclear stem cells were separated from the bone marrow immediately after the collection using Histopaque (10771, Sigma-Aldrich) and washed three times using phosphate buffered saline (PBS) (P493 SigmaAldrich). The number, viability, and expression of clusters of differentiation (CD) markers characterizing MSCs (CD44, CD90, CD105) were determined by flow 
cytometry (FACS Canto, Becton-Dickinson). Afterwards, adherent reticular shaped MSCs were cultured in a minimum essential medium (MEM) (56419C, SigmaAldrich), 10\%, fetal bovine serum (FBS) (F2442, Sigma-Aldrich), 1\%, PenicilinStreptomycin(P4333, Sigma-Aldrich) under tissue culture conditions at $37^{\circ} \mathrm{C}\left(5 \% \mathrm{CO}_{2}\right)$. During this cultivation MSCs acquired a surface expression of CD90 and CD105 which was not previously observable in bone marrow cells after the collection (Fig. 4). The medium was replaced every 3-4 days. Confluent MSCs were trypsinized (usually on day 10) using 0,05-0,25\% trypsin (59418C, Biosciences) and split into additional tissue culture flasks in order to maintain semi-confluent density. MSCs were harvested on day 17 of tissue culture expansion for the $1^{\text {st }}$ application (day 37 after the injury) into the injured tendon and repeated on day 40 of tissue culture expansion for the $2^{\text {nd }}$ application of MSCs (day 60 after the injury).

\section{$\underline{1 \text { st Application of MSCS }}$}

Preparation of MSCs: Cells were trypsinized on day 17 of tissue culture cultivation and washed in PBS, and analyzed for expresion of $\mathrm{CD}_{4} 4^{+}, \mathrm{CD}^{2} 0^{+}$, $\mathrm{CD}_{105^{+}}$by flow cytometry using monoclonal antibodies conjugated with fluorochromes (all purchased from Beckton-Dickinson). After centrifugation (1200 rpm/RT) cells were washed three times and resuspended in $2 \mathrm{ml}$ of physiological solution (B. Braun Melsungen AG). $1 \mathrm{ml}$ of resuspended cells was drawn into two screw-in-2ml-syringes (each containing $5 \times 10^{6}$ cells).

Preparation of injured tendon for application of MSCs: The flexor area of the distal metacarpus was shaved, cleaned and washed three times using betadine soap, and then carefully desinfected (three times- using betadine desinfection solution). MSCs were delivered into the injured SDFT on day 37 after the injury using two prepared syringes (above); the first puncture was applied palmarodorsalis and the second one lateromedialis. Afterwards the limb was covered by a three layer Robert-Johnson bandage.

\section{$\underline{2}^{\text {nd }}$ Application of MSCS}

The second application of MSCs was performed on day 60 after the injury in an analogous fashion (day 40 of tissue culture expansion).

\section{Rehabilitation Program}

After each application of the MSCs the horse was kept in it's stable, without movement, for three days. The following week the horse was engaged in moderate movement (walking) for about 15 minutes a day. After the first application of MSCs the horse was only walking. This movement was increased after the second application of MSCs by trotting for 2 minutes daily and then incrementally another 2 minutes of trotting were added in the following weeks. Starting on the 4 th week the horse was allowed to gallop for 2 minutes.

\section{Results}

Firstly, the injured tendon was examined using ultrasonographic evaluation with a 7.5 MHz linear probe (Fig. 1). By this means it was estimated that the lesion was approximately $70 \%$ of tendon tissue. The collection of bone marrow was carried out on the same day (day 20 after the injury), and mononuclear cells were separated on Histopaque and expanded ex vivo for three passages (see Materials and Methods). Under these tissue culture conditions MSCs (but not other cells) adhered and yielded a typical reticular morphology (Fig. 2). 


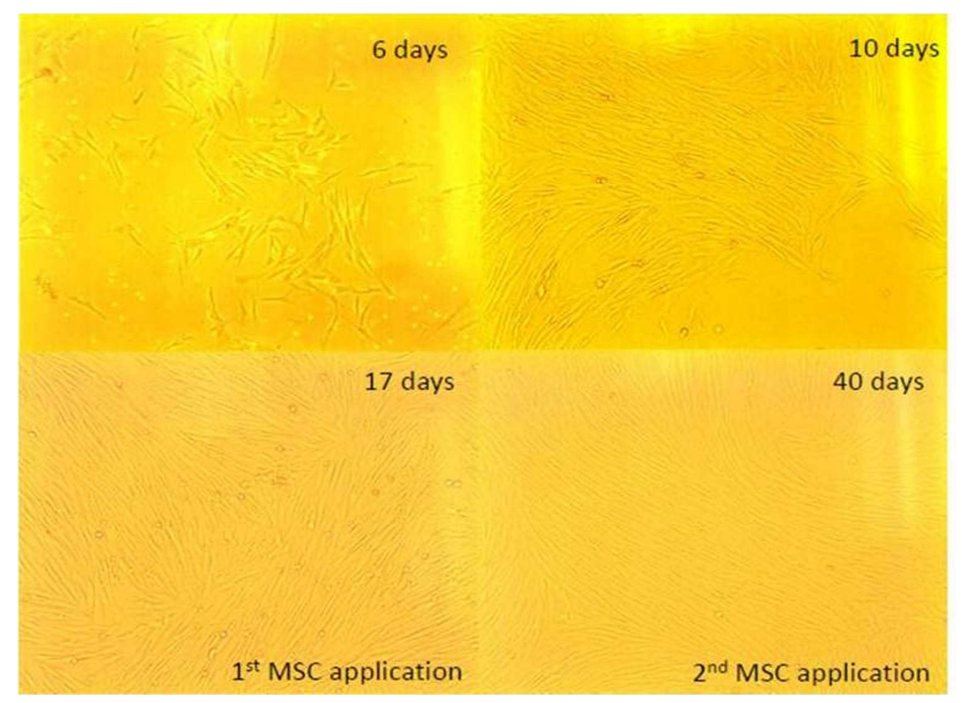

Fig. 2. Ex vivo Expansion of MSCs Collected from Bone Marrow on Day 20 after the Injury. These Cells were Cultivated in Tissue Culture for 6, 10, 17and 40 Days (Magnification 100x). Adherent Reticular Shaped MSCs Harvested on Day 17 and Day 40 were Used for the $1^{\text {st }}$ MSC Application or the $2^{\text {nd }}$ MSC Application into Injured Tendon, Respectively.

The majority of freshly isolated bone marrow derived mononuclear cells (day 0 of cultivation) expressed constitutively
CD44 marker and this marker exhibited an unaltered expression during cultivationon day 17 and 40 (Fig. 3).

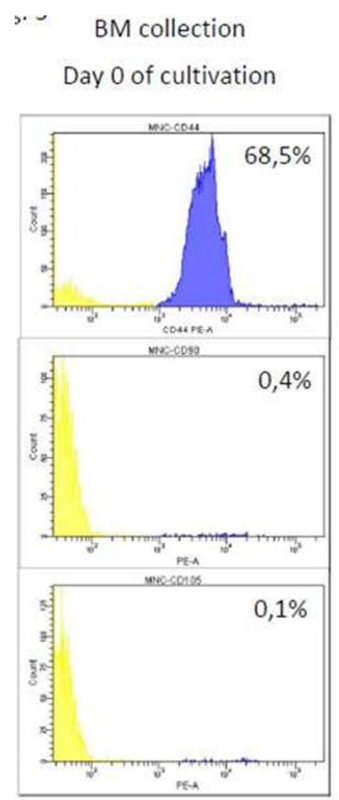

$1^{\text {st }}$ application of MSCS

Day 17 of cultivation
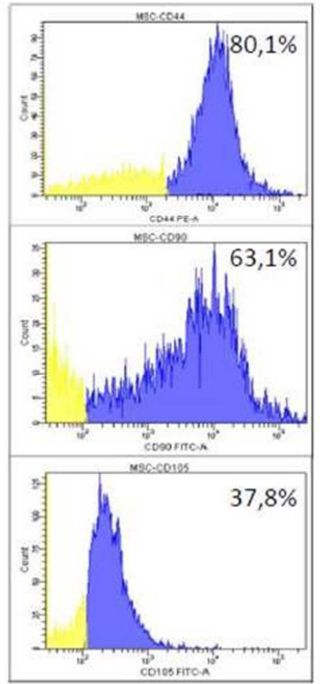

$2^{\text {nd }}$ application of MSCS

Day 40 of cultivation

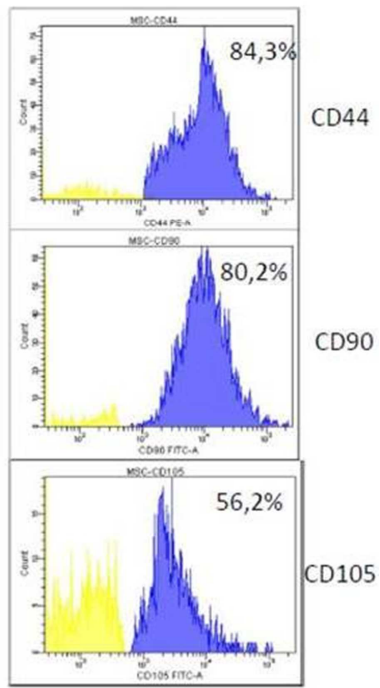

Fig. 3. Bone Marrow Mononuclear Cells Were Isolated; Analyzed for the Expression of CD44, CD90, and CD105 and Compared with their Expression on Ex Vivo Expanded MSCs on day 17 and Day 40 of Cultivationvia Flow Cytometry. Cells Without Expression of Any Marker are Depicted in Histograms as Yellow, While Cells Expressing Single Marker are Blue.

However, CD90 and CD105 expressions were observed on MSCs only later after cultivation on day 17 and day 40. These ex vivo expanded cells expressing CD90 and CD105 were harvested on day 17 of cultivation and used for the $1^{\text {st }}$ MSC 
application into the injured tendon. The $1^{\text {st }}$ application of MSCs was performed on day 37 after the injury using two punctures with syringes containing $5 \times 10^{6}$ cells each. The first puncture into the injured tendon was applied palmarodorsalis and the second one lateromedialis. The $2^{\text {nd }}$ application of MSCs was carried out on day 60 of the injury in an analogous fashion (Fig. 4).

$$
\text { day } 20 \quad \text { day } 60 \quad \text { day } 120
$$

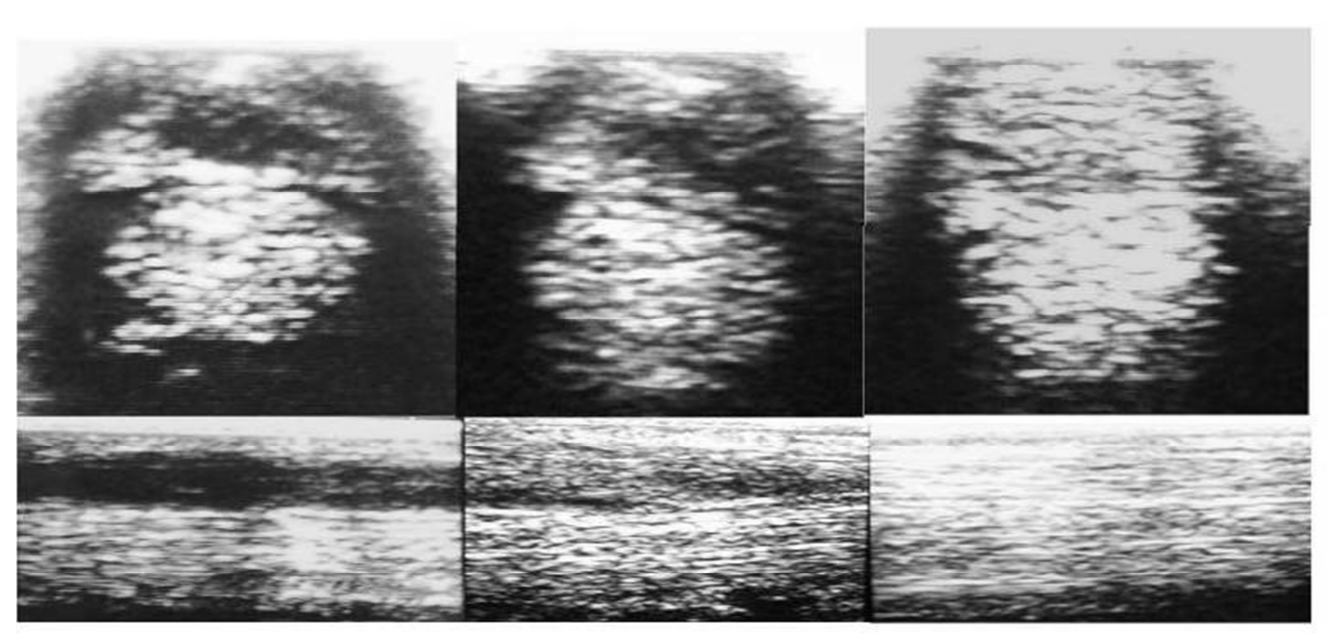

before treatment with MSCS

$2^{\text {nd }}$ application of MSCS

after treatment with MSCS

Fig. 4. Transversal (Top) and Longitudinal (Bottom) Projections of Ultrasound Examination of Distal Metacarpus of the Horse - Ultrasonograph on Day 20 (before Treatment with MSCs), Day 60 (after the $1^{\text {st }}$ and on the Day of the $2^{\text {nd }}$ Application of MSCs), and 120 Days after the Injury (after Treatment with MSCs).

The lesion of the tendon consisted of approximately $60 \%$ of healthy tissue and moderate improvement was observed. After the $2^{\text {nd }}$ application of MSCs the injured tendon was nearly completely healed (day 120 after the injury).

\section{Discussion}

Tendonitis is a very serious injury because it requires a long period of convalescence, which often creates new secondary tendon injuries above or below the origin alone. The aim of our therapy using MSCs was to ensure complete healing of the original tissue without additional scarring and to prevent the emergence of new secondary injuries caused by a lack of tendon elasticity [6]. MSCs can be harvested from various sources e.g. adipose tissue, umbilical cord blood, bone marrow, and peripheral blood $[7,8,9,10]$. In this study we chose bone marrow because it represents a well established source of MSCs $[8,15,16]$. MSCs from bone marrow were successfully collected from the tuber coxae of the horse as described above. The Tuber coxae was chosen over the sternum because of the ease with which the collection of bone marrow could proceed. Moreover, the tuber coxae did not show any signs of damage after the collection. Since MSCs adhere to plastic ex vivo expansion of bone marrow cells was used to enrich MSCs under these tissue culture conditions. The minimal criteria chosen for the characterization of equine MSCs were based on the MSC's adherence to the plastic when maintained in tissue culture and there is an increased expression of characteristic surface markers (CD44, CD90, CD105) [11, 12]. These ex vivo expanded MSCs used for therapy $\left(1^{\text {st }}\right.$ and $2^{\text {nd }}$ application) were maintained in tissue culture only fora limited number of passages (usually three passages) since the 
more passages the higher the risk of differentiation [9]. In this case we used a physiological solution for the delivery of MSCs into the injured tendon because damaged tissue was located in the middle of the SDFT and the risk of MSC release into the surrounding tissue was rather limited. Nevertheless, it is possible to retain MSCs using various scaffolds e.g. fibrinogen or hyaluronic acid. Notably, these scaffolds are mainly used for the regenerative cell therapy of bone fractures $[13,14]$. In some cases MSCs were applied only once and shortly after the injury [15]. In our study we attempted to facilitate the healing of tendon injuries by doubling the amount of MSCs, in order to accelerate the healing. In contrast to previous studies, we also used the $2^{\text {nd }}$ application of MSCs to improve delivery of expanded MSCs to the injured tendon. After the $2^{\text {nd }}$ application of MSCs (120 days after the injury) the horse resumed a complete training regime (March 2012) and four months later the horse participated in an international eventing competition in Strzegom (Poland) (May and June 2012). The therapy with ex vivo expanded MSCs allowed the horse to compete at the same level as it had done before the injury. Currently, the horse is without any health problems. In this case the healing of the injury after the transfer of MSCs was successful, however, so far we have treated only one horse with this type of injury. It will be necessary to treat several horses including a control group in order to prove beyond reasonable doubt that MSCs represent a breakthrough regenerative treatment. Such treatments are underway.

\section{References}

1. Georg, R., Maria, C., Gisela, A. \& Bianca, C. (2010). "Autologous Conditioned Plasma as Therapy of Tendon and Ligament Lesions in seven Horses," Journal of Veterinary Science, 11(2) 173175.

2. Becker, C. K., Savelberg, H. H., Buchner, H. H. \& Barneveld, A. (1998). "Long-term Consequences of Experimental Desmotomy of the Accessory Ligament of the Deep Digital Flexor Tendon in
Adult Horses," American Journal of Veterinary Research, 59(3)347-51.

3. Argüelles, D., Carmona, J. U., Climent, F., Munoz, E. \& Prades, M. (2008). "Autologous Platelet Concentrates as a Treatment for Musculoskeletal Lesions in Five Horses," Veterinary Record, 162 208-211.

4. Smith, R. K. W., Korda, M., Blunn, G. W. \& Goodship, A. E. (2003). "Isolation and Implantation of Autologous Equine Mesenchymal Stem Cells from Bone Marrow into the Superficial Digital Flexor Tendon as a Potential Novel Treatment," Equine Veterinary Journal, 35 99-102.

5. Bianco, P., Cao, X., Frenette, P. S., Mao, J. J., Robey, P. G., Simmons, P. J. \& Wang, C. Y. (2013). "The Meaning, the Sense and the Signifikance: Translating the Science of Mesenchymal Stem Cells into Medicine," Nature Medicine,19(1)35-42.

6. [6] Crovace, A., Lacitignola, L., Rossi, G. \& Francioso, E. (2010). "Histological and Immunohistochemical Evaluation of Autologous Cultured Bone Marrow Mesenchymal Stem Cells and Bone Marrow Mononucleated Cells in Collagenase-induced Tendinitis of Equine Superficial Digital Flexor Tendon," Veterinary Medicine International, 1-10.

7. de Mattos Carvalho, A., Alves, A. L., Golim, M. A., Moroz, A., Hussni, C. A., de Oliveira, P. G. \& Deffune, E. (2009). "Isolation and Immunophenotypic Characterization of Mesenchymal Stem Cells Derived from Equine Species Adipose Tissue," Veterinary Immunology and Immunopathology,132(2-4)303-6.

8. Richardson, L. E., Dudhia, J., Clegg, P. D. \& Smith, R. (2007). "Stem Cells in Veterinary Medicine - Attempts at Regenerating Equine Tendon after Injury," Trends in Biotechnology, 25(9)409-16.

9. Koerner, J., Nesic, D., Romero, J. D., Brehm, W., Mainil-Varlet, P. \& Grogan, S. 
P.(2006) "Equine Peripheral BloodDerived Progenitors in Comparison to Bone Marrow-Derived Mesenchymal Stem Cells," Stem Cells, 24(6)1613-9.

10.Koch, T. G., Heerkens, T., Thomsen, P. D. \& Betts, D. H. (2007). "Isolation of Mesenchymal Stem Cells from Equine Umbilical Cord Blood," BMC Biotechnology, 726.

11.Burk. J., Badylak, S. F., Kelly, J. \& Brehm, W. (2013). "Equine Cellular TherapyFrom Stall to Bench to Bedside?," Cytometry Part A, 83(1)103-13.

12.De Schauwer, C., Piepers, S., Van de Walle, G. R., Demeyere, K., Hoogewijs, M. K., Govaere, J. L., Braeckmans, K., Van Soom, A. \& Meyer, E. (2012). "In Search for Cross-Reactivity to Immunophenotype Equine Mesenchymal Stromal Cells By Multicolor Flow Cytometry," Cytometry Part A, 81(4)312-23.

13.Hale, B. W., Goodrich, L. R., Frisbie, D. D., McIlwraith, C. W. \& Kisiday, J. D. (2012). "Effect of Scaffold Dilution on Migration of Mesenchymal Stem Cells from Fibrin Hydrogels," American Journal of Veterinary Research, 73(2)313-8.

14.Kang, B. J., Ryu, H. H., Park, S. S., Kim, Y., Woo, H. M., Kim, W. H. \& Kweon, O. K. (2012). "Efect of Matrigel on the Osteogenic Potential of Canine Adipose Tissue- Derived Mesenchymal Stem Cells," The Journal of Veterinary Medical Science, 74(7)827-36.

15.Frisbie, D. D. \& Smith, R. K. W. (2010). "Clinical Update on the Use of Mesenchymal Stem Cells in Equine Orthopaedics," Equine Veterinary Journal, 42(1)86-9.

16.Delling, U., Lindner, K., Ribitsch, I., Jülke, H. \& Brehm, W. (2012). "Comparison of Bone Marrow Aspiration at the Sternum and the Tuber Coxae in Middle-aged Horses," Canadian Journal of Veterinary Research, 76(1) 52-56. 